\title{
miR-361-5p modulates metabolism and autophagy via the Sp1-mediated regulation of PKM2 in prostate cancer
}

\author{
ZHIXIN LING $^{1 *}$, DACHUANG LIU ${ }^{2,3 *}$, GUANGYUAN ZHANG ${ }^{1 *}$, \\ QING LIANG ${ }^{2}$, PING XIANG ${ }^{4}$, YAN XU $^{4}, \mathrm{CONGHUI} \mathrm{HAN}^{2}$ and TAO TAO $^{4}$ \\ ${ }^{1}$ Department of Urology, Zhongda Hospital, School of Medicine, Southeast University, Nanjing, Jiangsu 210009; \\ ${ }^{2}$ Department of Urology, Xuzhou Central Hospital, Xuzhou, Jiangsu 221009; ${ }^{3}$ Xuzhou Institute of \\ Medical Science, Xuzhou, Jiangsu 221009; ${ }^{4}$ Department of Urology, Anhui Provincial Hospital, \\ Anhui Medical University, Hefei, Anhui 230001, P.R. China
}

Received February 8, 2017; Accepted July 5, 2017

DOI: $10.3892 /$ or.2017.5852

\begin{abstract}
Prostate cancer (PCa) is a leading cause of death among men. The dysregulation of metabolism and autophagy contributes to the progression of $\mathrm{PCa}$. The transcription factor specificity protein 1 (Sp1) is implicated in the regulation of metabolism and autophagy. We confirmed that Sp1 is overexpressed in castration-resistant prostate cancer (CRPC) cells. However, the roles of Sp1 in PCa metabolism and autophagy remain unclear. Thus, in the present study, we retrieved the GSE35988 dataset from Gene Expression Omnibus (GEO) database to reinvestigate $\mathrm{Sp} 1$ expression and its role in $\mathrm{PCa} . \mathrm{We}$ found that in PCa, Spl knockdown significantly inhibited cell growth, aerobic glycolysis, and hypoxia-induced autophagy, which were accompanied by an increased G1 cell cycle arrest. Pearson correlation indicated that pyruvate kinase isoenzyme type M2 (PKM2) is positively correlated with Sp1 expression. Western blot analysis demonstrated that $\mathrm{Sp1}$ directly regulates PKM2; therefore, Sp1 modulates metabolism and autophagy in CRPC. Western blot analysis and luciferase reporter assay also indicated that the tumor suppressor miR-361-5p inversely regulates $\mathrm{Sp} 1$ by directly targeting the binding site in the 3'UTR of Sp1. miR-361-5p overexpression presented effects that are similar to Sp1 depletion in PCa. In summary, this study is the first to demonstrate that miR-361-5p suppresses the Sp1/PKM2 axis, consequently affecting the progression of PCa and the metabolism and autophagy of PCa cells.
\end{abstract}

Correspondence to: Dr Conghui Han, Department of Urology, Xuzhou Central Hospital, No. 199 Jiefang Road, Xuzhou, Jiangsu 221009, P.R. China

E-mail: conghuiseu@126.com

Dr Tao Tao, Department of Urology, Anhui Provincial Hospital, Anhui Medical University, No. 17 Luzhou Road, Hefei, Anhui 230001, P.R. China

E-mail: taotao860721@126.com

*Contributed equally

Key words: castration-resistant prostate cancer, miR-361-5p, specificity protein 1 , autophagy, pyruvate kinase isoenzyme type M2, metabolism
Therefore, targeting the miR-361-5p/Sp1/PKM2 pathway has considerable clinical significance in preventing the malignant progression of $\mathrm{PCa}$.

\section{Introduction}

Specificity protein $1(\mathrm{Sp} 1)$ is a transcription factor that binds to GC-rich motifs including GC-boxes, CACCC-boxes (also called GT-boxes), and basic transcription elements; Sp1 binds with high affinity and regulates the expression of numerous mammalian genes (1). Sp1 is an ideal target for cancer treatment given its important role in prostate cancer (PCa) progression, including cell proliferation, angiogenesis, differentiation, apoptosis, migration and invasion $(2,3)$. Recent evidence indicates that $\mathrm{Sp}$ transcription factors act in concert with Akt to regulate the abnormal metabolism of cancer cells (4). In addition, Sp1 inhibition significantly suppresses fatty acid synthase expression in PCa cells (5). However, the regulatory role of Sp1 in $\mathrm{PCa}$ cell metabolism remains unknown.

Autophagy promotes disease progression and therapeutic resistance in advanced PCa (6). Sp1 interacts with hypoxiainducible factor (HIF-1), transactivates vascular endothelial growth factor (VEGF), regulates the VEGF pathway and influences autophagy (7). PKM2, a modulator of glycolysis, also regulates the autophagic process by upregulating LC3B or Beclin-1 in glioma cells or in cancer-associated fibroblasts $(8,9)$. We speculate that autophagy in PCa may be modulated by the Sp1/PKM2 axis given that Sp1 regulates the transcriptional activity of PKM2 (10).

In this study, we illustrated that $\mathrm{Sp} 1$ plays a vital role in $\mathrm{PCa}$ metabolism and autophagy by positively regulating PKM2. In addition, miR-361-5p directly targets Sp1/PKM2 signaling, thereby modulating metabolism and autophagy in PCa.

\section{Materials and methods}

Data mining and bioinformatics analysis. The GSE35988 dataset was downloaded from GEO database (http://www. ncbi.nlm.nih.gov/geo) and re-analyzed with R (11). Pearson's correlation in MATLAB was used to analyze the relationship between SP1 and all identified genes $(\mathrm{P}<0.05)$. 
Cell culture. Two human PCa cell lines, namely, DU145 and PC3, were purchased from Nanjing KeyGen Biotech, Co., Ltd. (Nanjing, China). The cells were cultured at $37^{\circ} \mathrm{C}$ in a $20 \% \mathrm{O}_{2}$ humidified atmosphere with $5 \% \mathrm{CO}_{2}$ in RPMI-1640 medium that was supplemented with $10 \%$ fetal bovine serum (FBS; 100-700; Gemini Bio Products, Woodland, CA, USA), $50 \mathrm{U} / \mathrm{ml}$ penicillin and $50 \mathrm{mg} / \mathrm{ml}$ streptomycin (15140-122; Gibco Laboratories, Gaithersburg, MD, USA). Hypoxia experiments were performed in a hypoxic chamber (Coy Laboratory Products, Inc., Grass Lake, MI, USA) at $1 \% \mathrm{O}_{2}$ at $37^{\circ} \mathrm{C}$ in a $5 \%$ $\mathrm{CO}_{2}$ humidified environment.

Oligonucleotides, plasmid and cell transfection. Oligonucleotides were chemically synthesized by Shanghai GenePharma, Co., Ltd., (Shanghai, China), based on the following sequences: Sp1 siRNA, 5'-GGUCAGUUGGCAGA CUCUATT-3' (sense); siRNA negative control(NC), 5'-UUC UCCGAACGUGUCACGUTT-3' (sense); hsa-miR-361-5p mimics: 5'-ACCCCUGGAGAUUCUGAUAAUU-3'; negative control (NC): 5'-UUCUCCGAACGUGUCACGUTT-3'. Cells that were $60-70 \%$ confluent were transfected with Lipofectamine 2000 (11668-019; Invitrogen, Albuquerque, NM, USA). Oligonucleotides or plasmids formed transfection complexes with Lipofectamine 2000. The transfection complexes were then added to DU145 and PC 3 cells at different concentrations and incubated for 6-8 $\mathrm{h}$ before changing the medium.

RNA isolation and RT-qPCR. Total RNA was extracted from cells using TRIzol (15596018; Invitrogen) in accordance with the manufacturer's instructions. RNA concentration was measured using NanoDrop 2000c (Thermo Scientific, Wilmington, DE, USA). For miR-361-5p, the RT-qPCR reaction was performed with a HiScript RT kit (R132-01; Vazyme Biotech, Co., Ltd., Nanjing, China) and AceQ SYBR-Green PCR Master Mix (Q141-02/03; Vazyme Biotech) following the manufacturer's protocols. Primers were chemically synthesized by Nanjing Springen Biotech, Co., Ltd. (Nanjing, China). The miR-361-5p primers for amplification were as follows: stem-loop reverse transcription primer: 5'-CTCAACTGGT GTCGTGGAGTCGGCAATTCAGTTGAGGTACCCCT-3'; specific forward primer, 5'-ACACTCCAGCTGGGTTATC AGAATCTCC-3' and specific reverse primer: 5'-TGGTGTCG TGGAGTCG-3'. The U6 snRNA primers were as follows: forward: 5'-CTCGCTTCGGCAGCACA-3' and reverse, 5'-AA CGCTTCACGAATTTGCGT-3'. PCR was conducted with a $20 \mu \mathrm{l}$ reaction volume that contained $2 \mu \mathrm{l}$ of RT products, $1 \mu \mathrm{l}$ of $10 \mu \mathrm{M}$ miR-361-5p or U6 primer set, $10 \mu \mathrm{l}$ of $2 \mathrm{X}$ SYBR-Green PCR Master Mix, and $7 \mu \mathrm{l}$ of $\mathrm{ddH}_{2} \mathrm{O}$. Cycling conditions were as follows: $95^{\circ} \mathrm{C}$ for $3 \mathrm{~min}, 95^{\circ} \mathrm{C}$ for $15 \mathrm{sec}$, and $60^{\circ} \mathrm{C}$ for $60 \mathrm{sec}$. The last two steps were conducted with 40 cycles. RT-qPCR was performed using 7500 Real-Time PCR System (Applied Biosystems, Inc., Carlsbad, CA, USA). The gene expression threshold cycle (CT) values of miRNAs were calculated by normalizing to the internal control U6. Relative quantification values were calculated via the $2^{-\Delta \Delta \mathrm{Ct}}$ method (12).

Cell proliferation assay. DU145 and PC 3 cells were seeded in a 6-well plate and cultured overnight. The cells were transfected with oligonucleotides and cultured overnight. Subsequently, the cells were trypsinized and seeded at 2,000 cells/well (200 $\mu \mathrm{l} /$ well) in a 96 -well plate. At 24 -h post-transfection, cell proliferation was measured with a CCK-8 assay kit (KGA317; Nanjing KeyGen Biotech) in accordance with the manufacturer's instructions. Absorbance was detected at a wavelength of $450 \mathrm{~nm}$. Five wells were measured for cell viability in each treatment group. All independent treatments were performed in triplicate.

Cell cycle assay. Cell cycle distributions were analyzed using FACS (Becton-Dickinson, Franklin Lakes, NJ, USA). Cell cycle assay kits were procured from Nanjing KeyGen Biotech (KGA514). At $48 \mathrm{~h}$ post-transfection, cells were harvested and fixed with $75 \%$ ethanol at $-20^{\circ} \mathrm{C}$ overnight. Subsequently, the cells were incubated in $50 \mu \mathrm{g} / \mathrm{ml}$ propidium iodide and $1 \mathrm{mg} / \mathrm{ml}$ RNase for $30 \mathrm{~min}$ at room temperature and analyzed using flow cytometry. The experiments were performed in triplicate.

Metabolism assay. DU145 and PC3 cells were seeded in a 24-well plate that contained $200 \mu \mathrm{l}$ of media/well. To determine glucose and lactate levels in cells, the supernatants of the cell culture media were collected and assayed for glucose and lactate levels by using a glucose assay kit (K686; BioVision, Inc., San Francisco, CA, USA) and lactate assay kit (K627; BioVision), respectively, in accordance with the manufacturer's instructions. OD values of the supernatants were analyzed at $72 \mathrm{~h}$ after different treatments. The remaining glucose and lactate production were calculated based on the standard curve and normalized to the cell number.

Transmission electron microscopy (TEM). Cells were harvested 48 h post-transfection with Sp1 siRNA and NC siRNA. The samples were then pelleted and fixed in $2.5 \%$ glutaraldehyde (G5882; Sigma-Aldrich, St. Louis, MO, USA) in phosphate buffer ( $\mathrm{pH} 7.4$ ). Next, the samples were rinsed with phosphate buffer ( $\mathrm{pH} 7.4$ ) and postfixed with $2 \% \mathrm{OsO}_{4}$ (75633; Sigma-Aldrich) for $1 \mathrm{~h}$. The samples were then rinsed with water, dehydrated in a graded ethanol series [70\% ethanol (20 min), 96\% ethanol (20 min), and then $100 \%$ ethanol (2x20 min)] followed by propylene oxide (2x10 min) (SAJ-246210; Sigma-Aldrich), and maintained overnight in Epon 812 (45347-1L-F; Sigma-Aldrich). Subsequently, the samples were embedded in Epon 812 and then fumigated in a microwave oven at $60^{\circ} \mathrm{C}$. Ultrathin sections were obtained using Reichert Ultracut $\mathrm{E}$ microtome and then stained with uranyl acetate and lead citrate. Samples were imaged using an H-7650 transmission electron microscope (Hitachi, Ltd., Tokyo, Japan).

Western blot analysis. Radioimmunoprecipitation assay buffer (KGP701; Keygentec, Inc., Nanjing, China) was used to lyse cells for total proteins. Protein concentration was determined via the BCA method (KGP902; Keygentec). Protein samples (30 $\mu \mathrm{g}$ ) were electrophoresed on $10 \%$ sodium dodecyl sulphatepolyacrylamide electrophoresis gels (KGP113K; Keygentec) and transferred onto polyvinylidene fluoride membranes (IPVH00010; Millipore, Billerica, MA, USA). The membranes were blocked with $5 \%$ skim milk at room temperature for $1 \mathrm{~h}$ and incubated overnight at $4^{\circ} \mathrm{C}$ with diluted primary antibodies. Subsequently, the membranes were washed thrice 

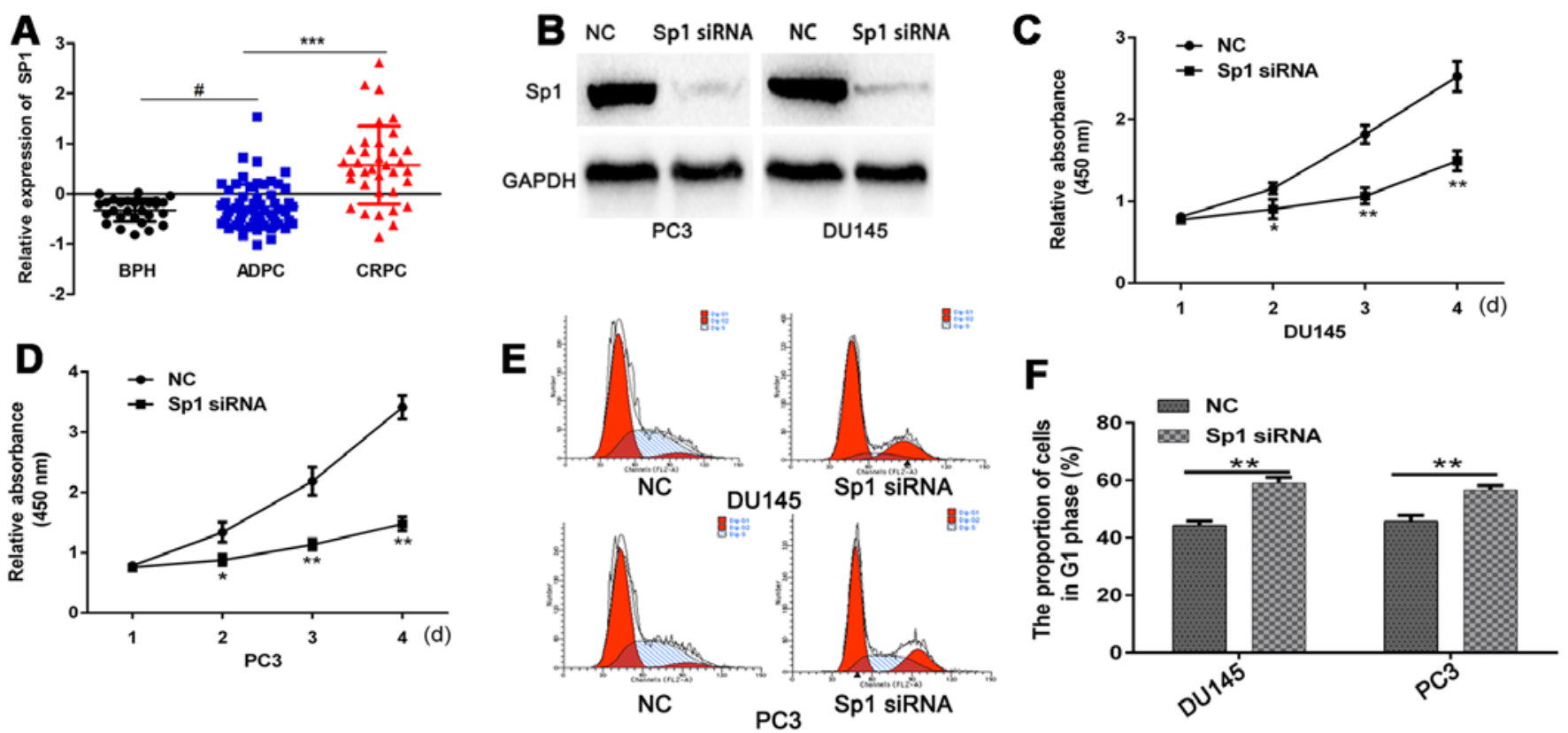

Figure 1. Knockdown of Sp1 inhibits cell proliferation and induces G1 phase arrest in CRPC. (A) Reanalysis of the GSE35988 dataset with R revealed that SP1 expression was significantly higher in CRPC than in ADPC. (B) Sp1 protein expression was silenced by transfecting DU145 and PC3 cells with Sp1 siRNA, as verified by western blot analysis. (C and D) CCK-8 assay revealed that the decreased Sp1 expression significantly decreased proliferative index of PC3 and DU145 cells. (E and F) Cell cycle assay conducted via flow cytometry showed that Sp1-depleted cells were arrested in G1 phase. ${ }^{*} \mathrm{P}<0.05,{ }^{* *} \mathrm{P}<0.01$.

with TBST buffer (pH 7.6, $20 \mathrm{mM}$ Tris-HCl, $137 \mathrm{mM} \mathrm{NaCl}$, $0.01 \%$ Tween-20) and then incubated with HRP-conjugated secondary antibodies at $37^{\circ} \mathrm{C}$ for $1 \mathrm{~h}$. After being washed with TBST buffer, the membranes were visualized using enhanced chemiluminescence ECL (WBKLS0050; Millipore). Protein levels were normalized to GAPDH levels. The related antibodies were HRP-conjugated monoclonal mouse anti-GAPDH (KC-5G5, 1:1,000; Shanghai Kangchen), rabbit polyclonal antiLC3B (18725-1-AP, 1:1,000; Proteintech Group, Inc., Chicago, IL, USA), rabbit monoclonal anti-Beclin-1 (3495, 1:1,000; Cell Signaling Technology, Danvers, MA, USA), rabbit monoclonal anti-PKM2 (4053, 1:1,000; Cell Signaling Technology), rabbit monoclonal anti-SP1 (9389S, 1:1,000; Cell Signaling Technology), HRP-labeled goat anti-rabbit secondary antibody (ZB-2301, 1:3000; Beijing Zhongshan Golden Bridge Biotechnology, Co., Ltd., Beijing, China).

Plasmid construction and luciferase reporter assay. The 3'-UTR segment of Sp1 mRNA with miR-361-5p binding site was amplified via PCR with human genomic DNA. To generate the Sp1 3'-UTR reporter, PCR products were cloned into the XhoI and NotI restriction sites that were downstream of the open reading frame of luciferase in the psiCHECK-2 $2^{\mathrm{TM}}$ Vector (c8021; Promega, Madison, WI, USA). Deleting the binding site for miR-361-5p generated the mutant reporter. For reporter assays, the cells were transfected with Sp1 3'UTR, mutant reporter plasmid and miR-361-5p mimics, or NC. Luciferase activity was measured by Dual-luciferase assays (E1910; Promega) $48 \mathrm{~h}$ after co-transfection.

Statistical analysis. Each value in this study was obtained from at least three independent experiments and presented as mean $\pm \mathrm{SD}$. The significance of differences was calculated using t-tests for two-group comparisons with the SPSS 22.0 (IBM Corp., Armonk, NY, USA). A probability of $\mathrm{P}<0.05$ was considered statistically significant.

\section{Results}

Sp1 affects castration-resistant prostate cancer (CRPC) in vitro. First, we used the GEO database to analyze the SP1 expression in benign prostatic hyperplasia tissues and $\mathrm{PCa}$ tissues at different stages. The results indicated that SP1 expression is significantly higher in CRPC than in androgendependent prostate cancer (ADPC) (Fig. 1A). To verify the function of Sp1 in CRPC, we transfected CRPC cells with Sp1 siRNA or NC. SP1 protein was detected by western blot analysis, and results showed that Sp1 siRNA efficiently silenced Sp1 gene expression (Fig. 1B). CCK-8 assays demonstrated that $\mathrm{Sp} 1$ depletion significantly decreased proliferative index of PC3 and DU145 cells compared with NC (Fig. 1C and D). Flow cytometry assay revealed that CRPC cells that were transfected with Sp1 siRNA demonstrated a higher rate of G1 cell cycle arrest (Fig. 1E and F). Moreover, Sp1 siRNA significantly decreased glucose consumption and lactate production in CRPC cells compared with those in the NC group (Fig. 2A and B).

Glycolysis positively regulates autophagy via various mechanisms (13). The transcription factor HIF-1 $\alpha$ is an important regulator of glycolysis, and its upregulation is the mechanism through which autophagy is induced under hypoxia or nutrient starvation $(14,15)$. Given that previous studies have indicated that decreased glycolysis decreases HIF-1 and inhibits autophagy (16), we investigated whether the inhibition of metabolism by Sp1 depletion affects hypoxiainduced autophagy in CRPC. 

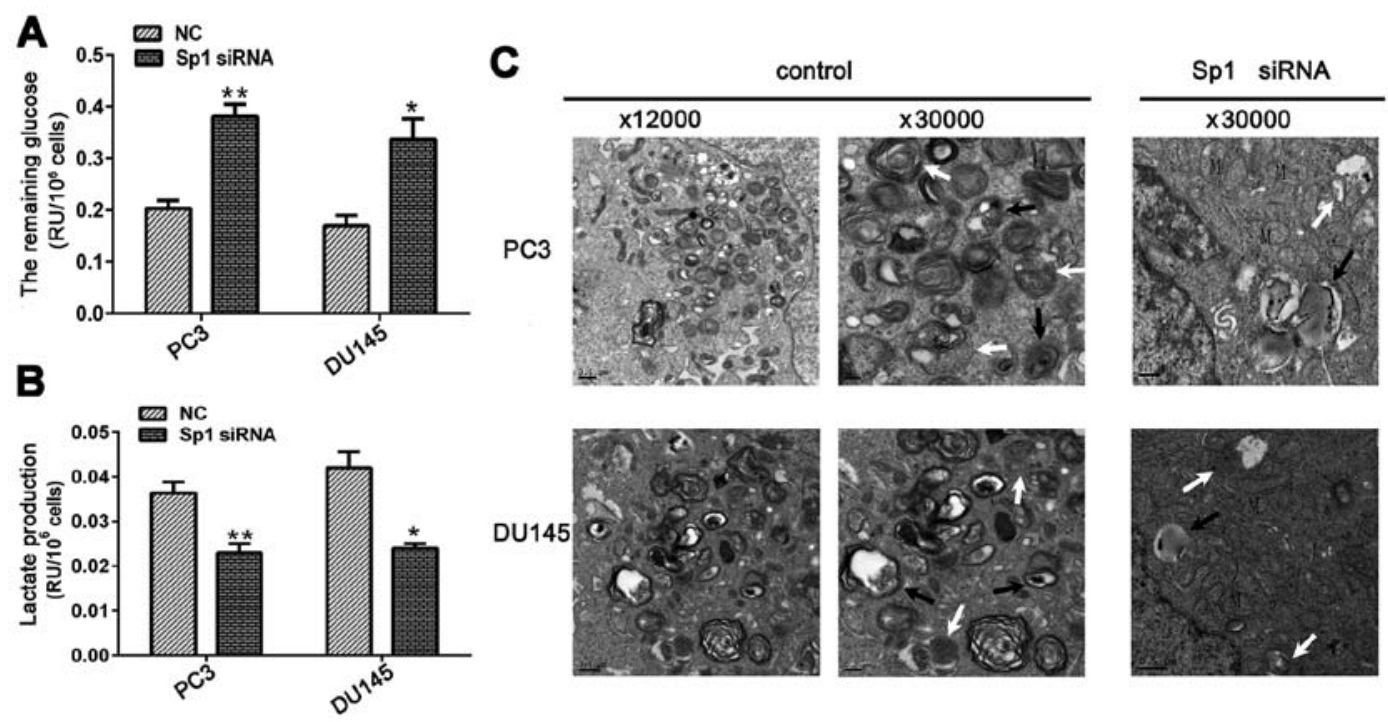

Figure 2. Silencing of Sp1 inhibits metabolism and hypoxia-induced autophagy in CRPC. (A) Supernatant of the culture medium was collected and subjected to glucose assay. (B) Supernatant of the culture medium was collected and subjected to lactate assay. (C) Transmission electron microscopy (TEM) images of PC3 and DU145 cells transfected with Sp1 siRNA or NC for $48 \mathrm{~h}$ under hypoxia. Images were captured at x12,000 and x30,000 magnifications. The white arrows indicate autophagosome (AP)-like structures, whereas the black arrows indicate autolysosome (AL)-like structures; M denotes mitochondria. Results are presented as mean \pm SD from three independent experiments. ${ }^{*} \mathrm{P}<0.05,{ }^{* *} \mathrm{P}<0.01$.

PC3 and DU145 cells were cultured at $37^{\circ} \mathrm{C}$ in $1 \% \mathrm{O}_{2}$ and $5 \% \mathrm{CO}_{2}$ humidified environment and were simultaneously transfected with Sp1 siRNA or NC. The cells were harvested for TEM at $48 \mathrm{~h}$ post-transfection. Autophagosomes, spherical structures with mono- or bilayer membranes, engulf degraded or abandoned organelles and deliver cytoplasmic components to lysosomes. As expected, hypoxic conditions significantly induced autophagy in PCa cells, as evidenced by large areas of autophagosomes or autolysosomes that were revealed by TEM. By contrast, Sp1 downregulation markedly inhibited hypoxia-induced autophagy, as indicated by the significantly decreased number of autophagosomes or autolysosomes in the Spl siRNA group in both CRPC cell lines (Fig. 2C).

Sp1 modulates metabolism and autophagy by directly targeting PKM2 in CRPC. To further evaluate the potential role of aberrantly expressed SP1, Pearson correlation was used to analyze the relationship between $\mathrm{Sp1}$ and all identified genes (Fig. 3A). The SP1-specific gene signature included 2,357 genes that are positively correlated with SP1 expression $(\mathrm{R}>0.4, \mathrm{P}<0.05)$ and 3,418 genes that are negatively correlated with $\mathrm{SP} 1$ expression $(\mathrm{R} \leq 0.4, \mathrm{P}<0.05)$ (data available upon request). Among these genes, a set of glycometabolism-related genes are positively correlated with SP1 expression. PKM2, a metabolic enzyme that executes the last step of aerobic glycolysis, is included in this set of genes, and its expression is significantly higher in CRPC than in androgen-dependent prostate cancer (Fig. 3B and C). Previous studies that identified Sp1 binding motifs in PKM2 promoter sequences have also asserted that Sp1 activates PKM2 promoter activity $(10,17)$; thus, western blot analysis was conducted in this study to confirm if Sp1 inhibition could decrease PKM2 expression. After $48 \mathrm{~h}$ of hypoxia experiments with Sp1 siRNA transfection, the cells were harvested for western blot analysis. Results indicated that transfection with Sp1 siRNA downregulated PKM2. Microtubule-associated protein 1 LC3, a specific marker of autophagy, is converted from LC3-I (inactivated form) to LC3-II (cleaved form) during autophagy. Western blot analysis showed that the conversion from LC3-I to LC3-II was inhibited in the Sp1 siRNA group, and that Beclin-1 expression also decreased (Fig. 3D). The signal ratio between LC3-II and LC3-I indirectly showed the extent of autophagy in PCa. The result indicated that the LC3-II/LC3-I ratio decreased in the Sp1 siRNA group and that autophagy decreased with Sp1 depletion (Fig. 3E).

Sp1 is a direct target of $m i R-361-5 p$. To identify the potential targets of miR-361-5p in PCa, bioinformatics analysis was performed with the website microRNA.org. Bioinformatics analysis revealed that miR-361-5p could bind to target sequences in the 3'UTR of Sp1 mRNA (Fig. 4A). Moreover, western blot analysis revealed that $\mathrm{Sp} 1$ protein expression was inhibited by treatment with miR-361-5p mimics (Fig. 4B). To confirm the direct miRNA-target interaction, Sp1 3'-UTR was cloned into a Dual-luciferase reporter cloning site in the psiCHECK-2 Dualluciferase vector. Luciferase activities remarkably decreased with increasing miR-361-5p levels (Fig. 4C).

miR-361-5p inhibits cell growth, glucose metabolism and autophagy in CRPC cells in vitro. RT-qPCR was utilized to examine miR-361-5p expression in cells at $48 \mathrm{~h}$ posttransfection with miR-361-5p mimics or NC into DU145 (PC3). miR-361-5p was expressed at higher levels in the miR-361-5p mimic group than in the NC group (Fig. 5A). Cell cycle analysis was conducted to determine the effect of miR-361-5p on cell proliferation. At $48 \mathrm{~h}$ post-transfection, the percentage of miR-361-5p-transfected cells in the G1 phase was higher than that of the NC-transfected cells (Fig. 5B). This result indicated that miR-361-5p induced G1 cell cycle arrest in CRPC cell lines. CCK-8 assays revealed that miR-361-5p overexpression significantly inhibited DU145 (PC3) proliferation (Fig. 5C and D). Moreover, miR-361-5p significantly 


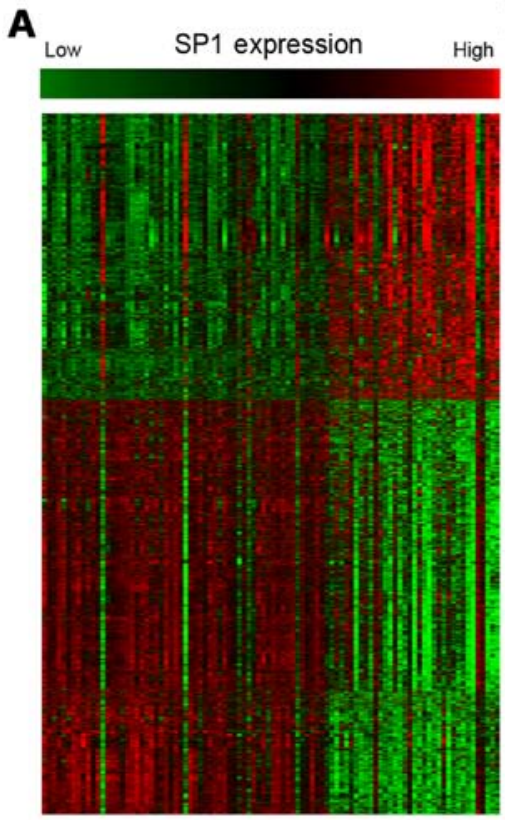

B

D
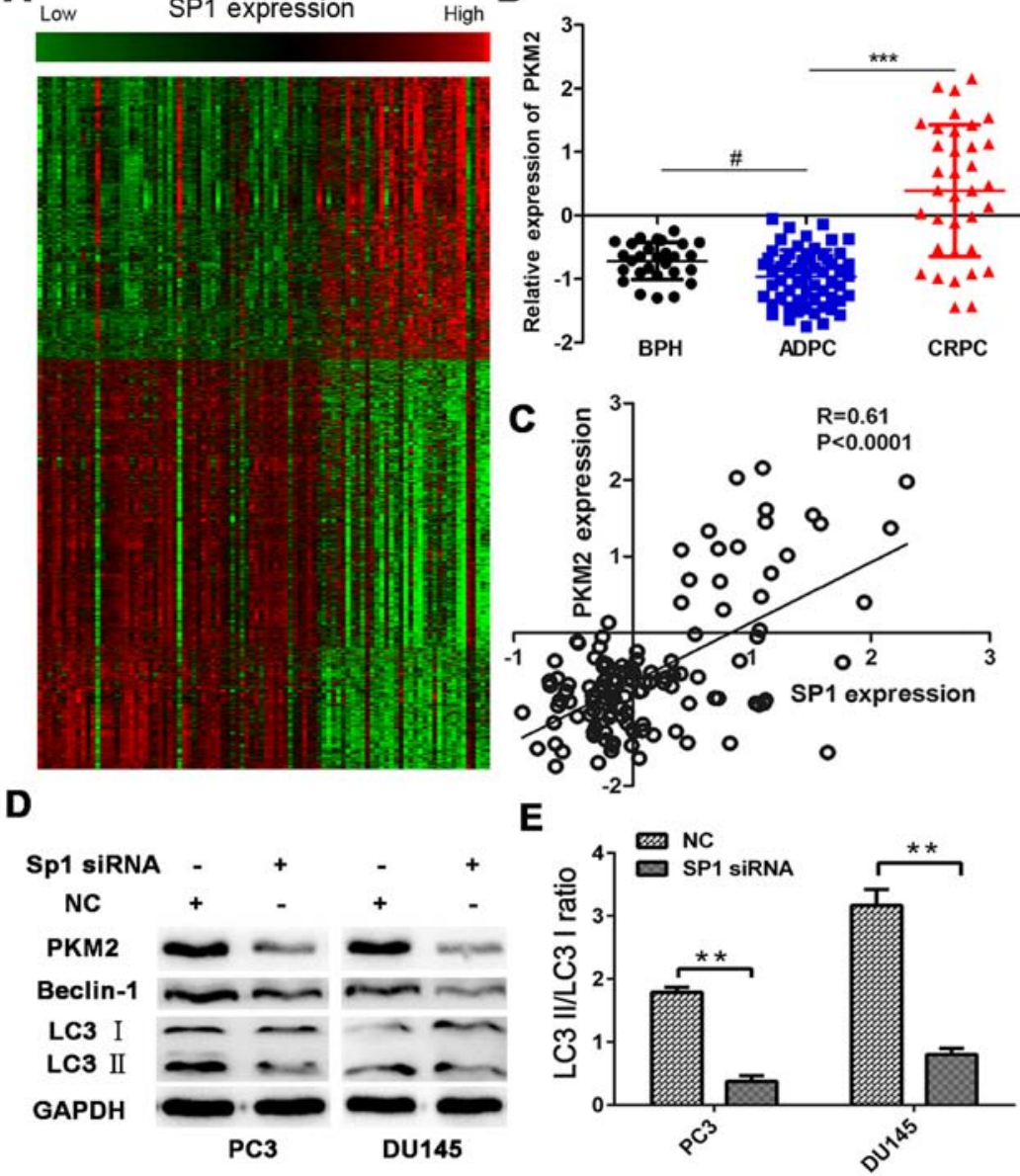

Figure 3. Sp1 modulates metabolism and autophagy by directly targeting PKM2 in CRPC. (A) Heat map of the derived gene expression signature correlated with Sp1 expression. The SP1-specific gene signature included 2,357 genes that are positively correlated with SP1 expression $(\mathrm{R}>0.4, \mathrm{P}<0.05)$ and 3,418 genes that are negatively correlated with SP1 expression $(\mathrm{R} \leq 0.4, \mathrm{P}<0.05)$. (B) PKM2 expression is significantly higher in CRPC than in ADPC via reanalysis of the GSE35988 dataset. (C) Pearson correlation analysis between SP1 and PKM2 in PCa. (D) Western blot analysis revealed that the expression levels of PKM2, Beclin-1 and LC3-II protein are lower in Sp1 siRNA-treated DU145 and PC3 cells than in the control group. (E) Band density of LC3-II normalized to LC3-I representing the relative autophagy of PCa. Compared with that in the control group, LC3-II/LC3-I ratio was significantly lower in Sp1-knockdown cells. Band density was measured using ImageJ software. Results are presented as mean $\pm \mathrm{SD}$ from three independent experiments. ${ }^{*} \mathrm{P}<0.05,{ }^{* * *} \mathrm{P}<0.01 .{ }^{* * * *} \mathrm{P}<0.01$.
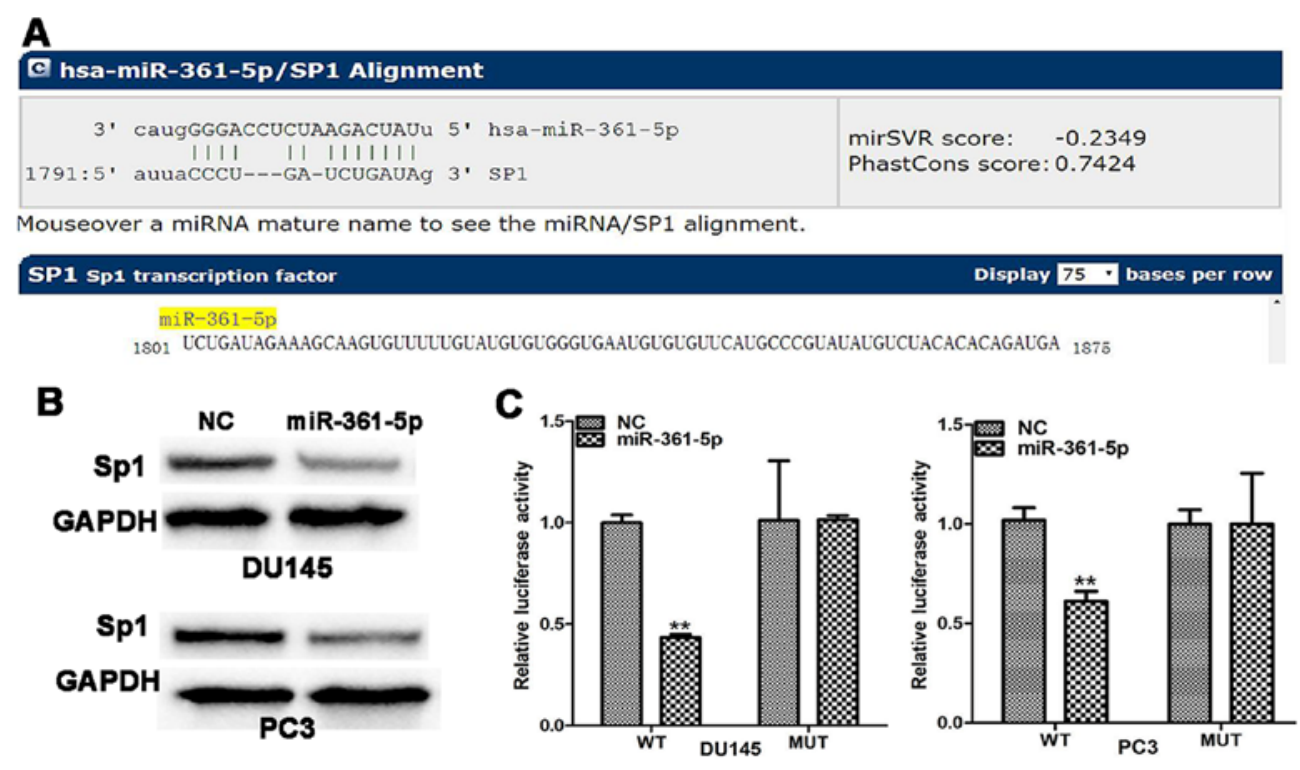

Figure 4. miR-361-5p directly targets Sp1. (A) microRNA.org showed that miR-361-5p could bind to target sequences that are located in the 3'UTR of Sp1 mRNA. (B) Western blot analysis revealed that Sp1 protein expression was inhibited by treatment with miR-361-5p mimics. (C) Sp1 3'UTR was cloned into a luciferase reporter cloning site in the psiCHECK-2 Dual-luciferase vector. Luciferase activities remarkably decreased with increasing miR-361-5p level. ${ }^{*} \mathrm{P}<0.05,{ }^{* *} \mathrm{P}<0.01$. 

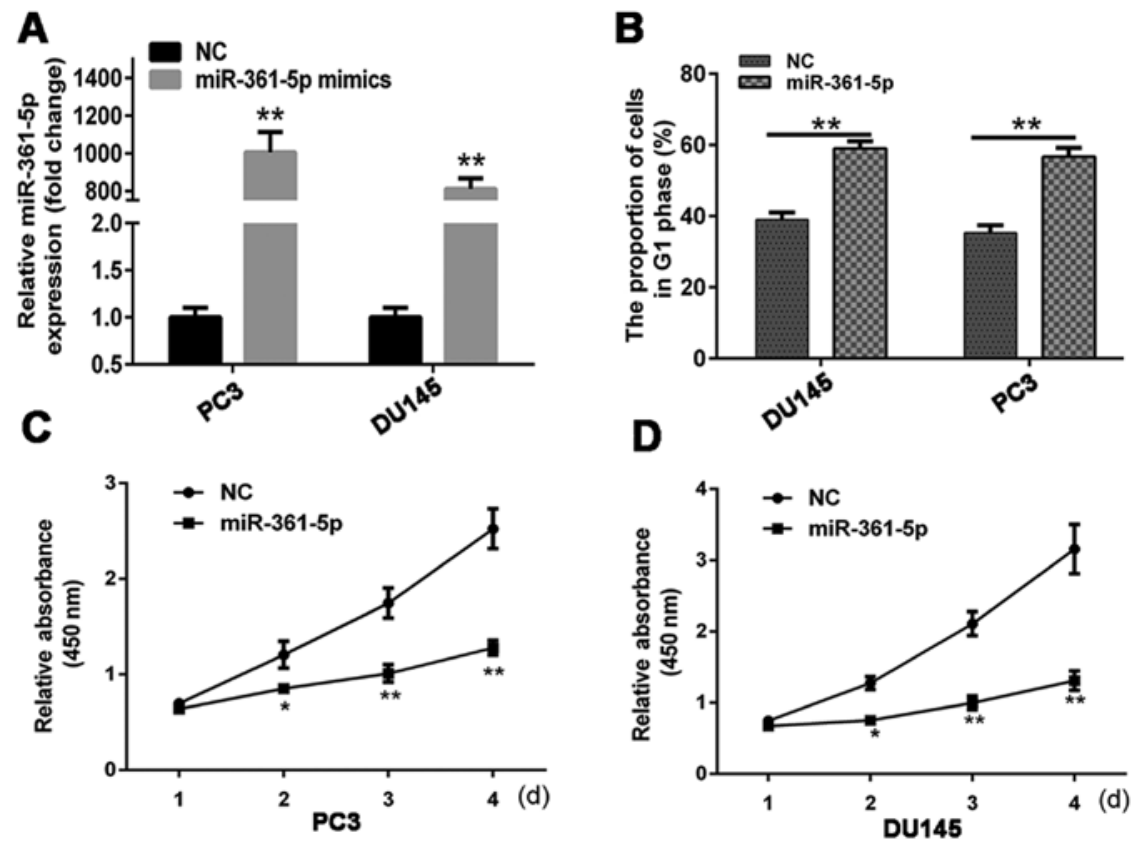

Figure 5. miR-361-5p suppresses cell growth and induces cell cycle arrest in CRPC cells. (A) RT-qPCR showed that transfection with miR-361-5p mimics increased miR-361-5p expression compared with transfection with NC. (B) Cells that ectopically expressed miR-361-5p were arrested in G1 phase. (C and D) CCK-8 assays revealed that miR-361-5p overexpression significantly inhibited cell proliferation. ${ }^{*} \mathrm{P}<0.05,{ }^{* *} \mathrm{P}<0.01$.

A
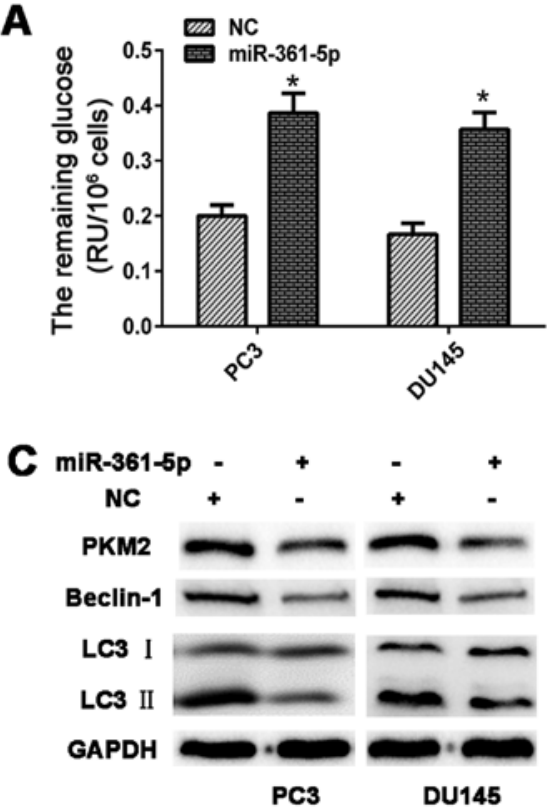

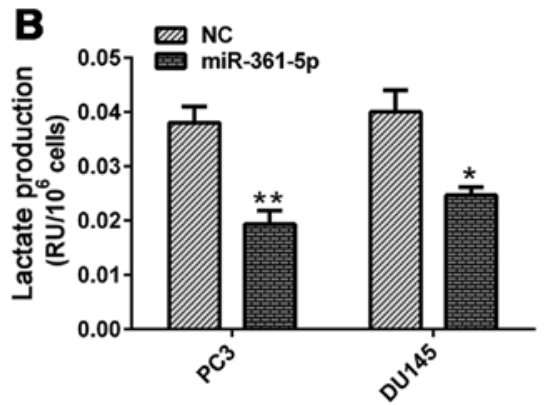

D

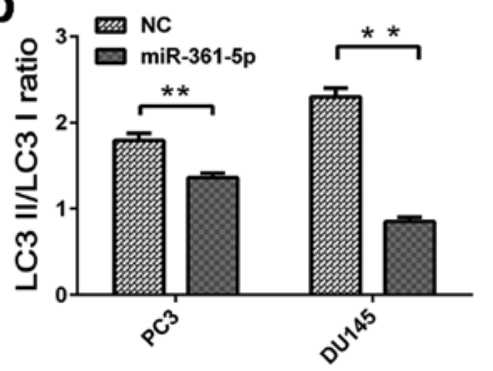

Figure 6. miR-361-5p regulates glucose metabolism and autophagy in CRPC cells. (A and B) Glucose consumption and lactate production were measured with a glucose assay kit and a lactate assay kit, respectively. (C and D) Expression levels of PKM2, Beclin-1 and LC3-II protein were analyzed by western blot analysis. The LC3-II/LC3-I ratio was calculated as that relative band density as measured by ImageJ software. Results are presented as mean \pm SD from three independent experiments. ${ }^{*} \mathrm{P}<0.05,{ }^{* *} \mathrm{P}<0.01$.

reduced glucose consumption and lactate production in the CRPC cells relative to that in the $\mathrm{NC}$ group (Fig. 6A and B).

miR-361-5p mimics or NC were transfected into CRPC cells, which were cultured under hypoxic conditions. The cells were harvested for western blot analysis at $48 \mathrm{~h}$ post-transfection. miR-361-5p overexpression significantly decreased PKM2, Beclin-1 and LC3B expression levels. Moreover, the LC3-II/LC3-I ratio of the miR-361-5p group was also lower than that in the NC group, indicating that miR-361-5p negatively regulates autophagy in PCa cells (Fig. 6C and D).

\section{Discussion}

Altered energy metabolism is proving to be as widespread in cancer cells as many of the other cancer-associated traits that have been accepted as cancer hallmarks. Previous studies 
have reported that sarcosine induces invasion and intravasation via elevated metabolism in an in vivo PCa model (18), and that androgens regulate PCa cell growth via an AMPKPGC-1 $\alpha$-mediated metabolic switch (19). Autophagy can meet the increased nutritional demand of proliferative cancer cells and is responsible for resistance to therapies, including radiotherapy, chemotherapy and androgen deprivation, in PCa (20-22). Recent findings also indicated that the increased expression of autophagy protein is associated with a high Gleason score and extraprostatic invasion (23). Therefore, targeting metabolism and autophagy could be a novel route for managing CRPC.

$\mathrm{Sp} 1$ is a transcription factor that is an essential target for regulating the translation rates and additional metabolic processes of cell growth $(17,24,25)$. Consistent with a previously reported finding that $\mathrm{Sp} 1$ is upregulated in numerous cancers with poor prognosis (3), we demonstrated that Sp1 upregulation is common in CRPC tissues and further explored the roles of Sp1 in CRPC. After determining that Sp1 expression increased in CRPC, we further investigated the functions and underlying mechanisms of Spl by inhibiting its expression.

The present study indicated that Sp1 depletion inhibits cell growth, metabolism and autophagy in CRPC. Western blot analysis revealed that suppressing $\mathrm{Sp} 1$ decreased the expression levels of PKM2 and of the autophagy markers Beclin-1 and LC3B-II in PCa cells that were cultured under hypoxia. A previous study (26) reported that five putative binding regions for $\mathrm{Sp} 1$ in the PKM promoter region and the dephosphorylation of Sp1 can enhance DNA-binding activity and increase the expression of PKM2 (16). We believe that Sp1 regulates PKM2 at the transcriptional level, thus modulating metabolism and cellular growth in PCa. In view of the interplay between metabolism and autophagy, the downregulation of PKM2 induces autophagy impairment in 2fTGH cells $(27,28)$. HIF-1 $\alpha$-induced autophagy promotes tumor progression (29). PKM2 acts as a HIF-1 $\alpha$ co-activator to promote Warburg effects in cancer cells and the PKM2 downregulation impairs autophagy (30). Our results illustrated that Sp1 plays a vital role in PCa cell metabolism and autophagy by positively regulating PKM2.

We have previously proved that miR-361-5p was downregulated and played a tumor suppressor role in CRPC (31), and we further illustrated that miR-361-5p was involved in the regulation of autophagy and metabolism in CRPC. Moreover, we showed that Sp1 was highly expressed and could modulate autophagy and metabolism in CRPC. Via bioinformatics analysis, it revealed that $\mathrm{miR}-361-5 \mathrm{p}$ could bind to target sequences in the 3'UTR of Sp1 mRNA. Then, we further explored whether miR-361-5p could regulate autophagy and metabolism via inverse regulation of $\mathrm{Sp} 1$. Western blot analysis and luciferase reporter assay demonstrated that miR-361-5p can reversely regulate $\mathrm{Sp} 1$ by directly targeting the mRNA of $\mathrm{Sp} 1$, as predicted by bioinformatics analysis. miR-361-5p overexpression in PCa cell lines presented biological functions that are similar to those of Sp1 depletion; thus, miR-361-5p modulates cell proliferation, metabolism and autophagy in $\mathrm{PCa}$ in an Sp1-dependent manner.

In summary, our findings revealed that miR-361-5p is a tumor suppressor. By contrast, $\mathrm{Sp1}$ is an oncogene as it positively regulates PKM2 in PCa. Notably, miR-361-5p inhibits
CRPC cell proliferation, metabolism, and autophagy by directly targeting Sp1/PKM2 signaling, which is a potential target in $\mathrm{PCa}$ therapy.

\section{Acknowledgements}

The present study was supported by the National Natural Science Foundation of China (81272557), the Natural Science Foundation of Jiangsu Province (BK20151158), the Talent Peak Project of Jiangsu Province (2014-WSW-067), the Innovation Team Project of Xuzhou Central Hospital(XZB201610), the Fundamental Research Funds for the Central Universities and the Scientific Research Innovation Program for College and University Graduates of Jiangsu Province(KYZZ16_0135), and the Natural Science Foundation of Anhui Province (1708085QH202).

\section{References}

1. Imataka H, Sogawa K, Yasumoto K, Kikuchi Y, Sasano K, Kobayashi A, Hayami M and Fujii-Kuriyama Y: Two regulatory proteins that bind to the basic transcription element (BTE), a GC box sequence in the promoter region of the rat P-4501A1 gene. EMBO J 11: 3663-3671, 1992.

2. Black AR, Black JD and Azizkhan-Clifford J: Sp1 and krüppellike factor family of transcription factors in cell growth regulation and cancer. J Cell Physiol 188: 143-160, 2001.

3. Sankpal UT, Goodison S, Abdelrahim M and Basha R: Targeting $\mathrm{Sp} 1$ transcription factors in prostate cancer therapy. Med Chem 7: 518-525, 2011.

4. Archer MC: Role of sp transcription factors in the regulation of cancer cell metabolism. Genes Cancer 2: 712-719, 2011.

5. Lu S and Archer MC: Sp1 coordinately regulates de novo lipogenesis and proliferation in cancer cells. Int J Cancer 126: 416-425, 2010.

6. Farrow JM, Yang JC and Evans CP: Autophagy as a modulator and target in prostate cancer. Nat Rev Urol 11: 508-516, 2014.

7. Alam H, Maizels ET, Park Y, Ghaey S, Feiger ZJ, Chandel NS and Hunzicker-Dunn M: Follicle-stimulating hormone activation of hypoxia-inducible factor-1 by the phosphatidylinositol 3-kinase/ AKT/Ras homolog enriched in brain (Rheb)/mammalian target of rapamycin (mTOR) pathway is necessary for induction of select protein markers of follicular differentiation. J Biol Chem 279: 19431-19440, 2004.

8. Chiavarina B, Whitaker-Menezes D, Martinez-Outschoorn UE, Witkiewicz AK, Birbe R, Howell A, Pestell RG, Smith J, Daniel R, Sotgia F, et al: Pyruvate kinase expression (PKM1 and PKM2) in cancer-associated fibroblasts drives stromal nutrient production and tumor growth. Cancer Biol Ther 12: 1101-1113, 2011.

9. Ahmad F, Dixit D, Joshi SD and Sen E: G9a inhibition induced PKM2 regulates autophagic responses. Int J Biochem Cell Biol 78: 87-95, 2016.

10. Yang W and Lu Z: Regulation and function of pyruvate kinase M2 in cancer. Cancer Lett 339: 153-158, 2013.

11. Grasso CS, Wu YM, Robinson DR, Cao X, Dhanasekaran SM, Khan AP, Quist MJ, Jing X, Lonigro RJ, Brenner JC, et al: The mutational landscape of lethal castration-resistant prostate cancer. Nature 487: 239-243, 2012

12. Livak KJ and Schmittgen TD: Analysis of relative gene expression data using real-time quantitative PCR and the 2(-Delta Delta C(T)) method. Methods 25: 402-408, 2001.

13. Eng $\mathrm{CH}$ and Abraham RT: The autophagy conundrum in cancer: Influence of tumorigenic metabolic reprogramming. Oncogene 30: 4687-4696, 2011.

14. Peng X, Gong F, Chen Y, Jiang Y, Liu J, Yu M, Zhang S, Wang M, Xiao $\mathrm{G}$ and Liao $\mathrm{H}$ : Autophagy promotes paclitaxel resistance of cervical cancer cells: Involvement of Warburg effect activated hypoxia-induced factor 1- $\alpha$-mediated signaling. Cell Death Dis 5: e1367, 2014.

15. Sun Y, Xing X, Liu Q, Wang Z, Xin Y, Zhang P, Hu C and Liu Y: Hypoxia-induced autophagy reduces radiosensitivity by the HIF-1 $\alpha / \mathrm{miR}-210 / \mathrm{Bcl}-2$ pathway in colon cancer cells. Int J Oncol 46: 750-756, 2015. 
16. Schäfer D, Hamm-Künzelmann B and Brand K: Glucose regulates the promoter activity of aldolase $\mathrm{A}$ and pyruvate kinase M2 via dephosphorylation of Sp1. FEBS Lett 417: 325-328, 1997.

17. Discher DJ, Bishopric NH, Wu X, Peterson CA and Webster KA: Hypoxia regulates beta-enolase and pyruvate kinase-M promoters by modulating Sp1/Sp3 binding to a conserved GC element. J Biol Chem 273: 26087-26093, 1998.

18. Khan AP, Rajendiran TM, Ateeq B, Asangani IA, Athanikar JN, Yocum AK, Mehra R, Siddiqui J, Palapattu G, Wei JT, et al: The role of sarcosine metabolism in prostate cancer progression. Neoplasia 15: 491-501, 2013.

19. Tennakoon JB, Shi Y, Han JJ, Tsouko E, White MA, Burns AR, Zhang A, Xia X, Ilkayeva OR, Xin L, et al: Androgens regulate prostate cancer cell growth via an AMPK-PGC-1 $\alpha$-mediated metabolic switch. Oncogene 33: 5251-5261, 2014.

20. Cao C, Subhawong T, Albert JM, Kim KW, Geng L, Sekhar KR, $\mathrm{Gi}$ YJ and Lu B: Inhibition of mammalian target of rapamycin or apoptotic pathway induces autophagy and radiosensitizes PTEN null prostate cancer cells. Cancer Res 66: 10040-10047, 2006.

21. Bennett HL, Stockley J, Fleming JT, Mandal R, O'Prey J, Ryan KM, Robson CN and Leung HY: Does androgen-ablation therapy (AAT) associated autophagy have a pro-survival effect in LNCaP human prostate cancer cells? BJU Int 111: 672-682, 2013.

22. Li M, Jiang X, Liu D, Na Y, Gao GF and Xi Z: Autophagy protects $\mathrm{LNCaP}$ cells under androgen deprivation conditions. Autophagy 4: 54-60, 2008.

23. Giatromanolaki A, Sivridis E, Mendrinos S, Koutsopoulos AV and Koukourakis MI: Autophagy proteins in prostate cancer: relation with anaerobic metabolism and Gleason score. Uro Oncol 32: 39.e11-38, 2014.
24. Wong N, De Melo J and Tang D: PKM2, a central point of regulation in cancer metabolism. Int J Cell Biol 2013: 242513, 2013

25. Siegel R, Ma J, Zou Z and Jemal A: Cancer statistics, 2014. CA Cancer J Clin 64: 9-29, 2014.

26. Netzker R, Weigert $C$ and Brand K: Role of the stimulatory proteins $\mathrm{Sp} 1$ and $\mathrm{Sp} 3$ in the regulation of transcription of the rat pyruvate kinase M gene. Eur J Biochem 245: 174-181, 1997.

27. Jiang M: Interplay between autophagy and metabolism in Ras mutation-induced tumorigenesis. Asian J Androl 13: 610-611, 2011.

28. Altuntas S, Rossin F, Marsella C, D'Eletto M, Diaz-Hidalgo L, Farrace MG, Campanella M, Antonioli M, Fimia GM and Piacentini M: The transglutaminase type 2 and pyruvate kinase isoenzyme M2 interplay in autophagy regulation. Oncotarget 6: 44941-44954, 2015.

29. Bellot G, Garcia-Medina R, Gounon P, Chiche J, Roux D, Pouysségur J and Mazure NM: Hypoxia-induced autophagy is mediated through hypoxia-inducible factor induction of BNIP3 and BNIP3L via their BH3 domains. Mol Cell Biol 29: 2570-2581, 2009.

30. Luo W, Hu H, Chang R, Zhong J, Knabel M, O'Meally R, Cole RN, Pandey A and Semenza GL: Pyruvate kinase M2 is a PHD3-stimulated coactivator for hypoxia-inducible factor 1 . Cell 145: 732-744, 2011

31. Liu D, Tao T, Xu B, Chen S, Liu C, Zhang L, Lu K, Huang Y, Jiang L, Zhang X, et al: MiR-361-5p acts as a tumor suppressor in prostate cancer by targeting signal transducer and activator of transcription-6 (STAT6). Biochem Biophys Res Commun 445: 151-156, 2014. 